RECEPTOR LIPIDS

GM1 gets sorted

Dev. Cell 23, 573-586 (2012)

The ganglioside GM1 acts as a lipid-based receptor for internalization and traffic of cholera toxin (CT), which is ultimately sorted to the endoplasmic reticulum of host cells. As with other acidic glycosphingolipids, both the extracellular oligosaccharide and the membraneanchoring ceramide of GM1 have rich structural diversity. Because different GM1 species have different capacities to sort CT to the endoplasmic reticulum, Chinnapen et al. set out to determine whether this was due to the specific ceramide structures. The authors synthesized five different GM1 structures, differing only in the fatty acyl chains in the ceramide domain, and monitored their trafficking in the presence and absence of CT in live cells by fluorescence microscopy. They found that sorting of the different GM1 species from the plasma membrane ultimately to the endoplasmic reticulum or from the plasma membrane to late endosomes is dictated by the ceramide domain. Only the short-chain and the unsaturated species trafficked retrograde into the endoplasmic reticulum, suggesting that these species are the functional CT receptors. Indeed, only these GM1 species led to downstream signaling. Further experiments examining the effect of $\mathrm{CT}$ binding on GM1 traffic and of disruption of rafts revealed that a lipid-raft mechanism is most likely involved in GM1 sorting at steps leading into the retrograde endoplasmic reticulum-directed pathway. Therefore, both major tenets of lipid sorting-the 'molecular shape' and 'lipid raft' hypotheses-regulate GM1 and CT traffic.

are key members of a previously undetected surveillance system that is responsible for degrading incompletely capped mRNAs. JMF

BIOSYNTHESIS

\section{Acting in self-defense}

Angew. Chem. Int. Ed. Engl., published online 17 September 2012; doi:10.1002/anie. 201204109

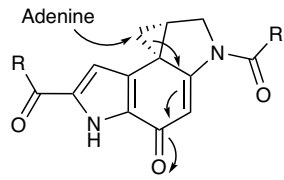

Natural product biosynthetic clusters often include efflux pumps or other enzymes that provide resistance to these frequently toxic compounds. The gene cluster for the DNAalkylating agent yatakemycin contains five genes, $y t k R 2-y t k R 6$, that could have a role in resistance: whereas YtkR6 is homologous to drug-resistance transporters, YtkR2-YtkR5 are homologous to enzymes involved in baseexcision repair, whereby damaged DNA bases are first cleaved by DNA glycosylases to reveal an abasic site and then further repaired to regenerate the parent DNA sequence. To test whether this mechanism might be operative in bacteria synthesizing yatakemycin, $\mathrm{Xu}$ et al. focused on YtkR2, the putative DNA glycosyltransferase. Inactivation of this gene in Streptomyces sp. TP-A0356 resulted in decreased production of the natural product, whereas overexpression of the gene increased production, supporting a functional role for the enzyme in protecting cells from yatakemycin. The enzyme was also able to protect Escherichia coli cells from yatakemycin as well as another alkyating agent. In vitro assays confirmed that the purified enzyme yielded an adenine-modified yatakemycin, with mutation of residues known to be important for glycosyltransferase function as well as those proposed to be involved in yatakemycin recognition reducing YtkR2 activity. These results highlight a new self-resistance mechanism and raise new questions regarding DNA glycosylase substrate specificity.

$C G$

PROTEASOME INHIBITION

\section{Missing amino acids}

Mol. Cell, published online 5 September 2012; doi:10.1016/j.molcell.2012.08.003

The proteasome degrades many cellular proteins, and proteasome inhibitors induce cancer cell death, but the mechanism underlying inhibitor-mediated death is not known. Suraweera et al. now show that temperature-sensitive proteasome inhibition in yeast leads to a decrease in the amount of free amino acids and that supplementation of proteasome-inhibited yeast cells with amino acids can rescue the lethality associated with proteasome inhibition without promoting proteasome activity. The authors also showed that treatment of mammalian cells with small-molecule proteasome inhibitors led to a decrease in the amount of select amino acids, including cysteine, asparagine and aspartate. Amino acid supplementation increased cell survival in a dose-dependent manner in response to inhibitors without rescuing either proteasome activity or blocking inhibitor function. Proteasome inhibitors induce the integrated stress response and autophagy in treated cells, and cysteine supplementation was sufficient to decrease the induction of these pathways. Blocking amino acid consumption through protein synthesis with cyclohexamide was sufficient to counter the drop in the amount of amino acids and to decrease inhibitor-induced lethality. Inhibitor-induced amino acid shortage was also observed in Drosophila, and amino acid supplementation could rescue lethality in treated flies. Taken together, these data indicate that amino acid scarcity is the signal that leads to stress responses and ultimately cell death in multiple species.

\section{BIOMATERIALS}

\section{In living color}

Angew. Chem. Int. Ed. Engl., published online 5 September 2012; doi:10.1002/anie.201205336

Proc. Natl. Acad. Sci. USA, published online 10 September 2012; doi:10.1073/pnas.1210105109

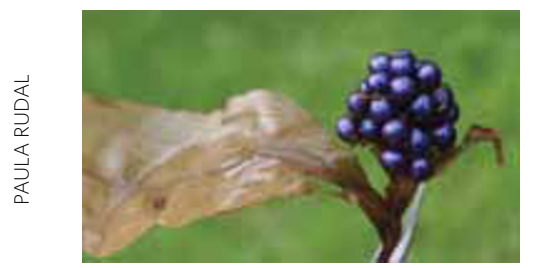

Color is abundant in the natural world, as are the mechanisms used to create it. Two recent studies explore the basis for the iridescent sheens in fish and plants. Gur et al. focused on Cyprinus carpio, or Japanese koi. Though it is known that the coloration of many fish is due to the presence of anhydrous guanine crystal arrays and that other organisms contain stable layers of amorphous guanine, there was no prior demonstration within biological systems that an organic molecule could pass through an amorphous phase en route to a crystalline phase. The authors used cryo-SEM imaging, microspot X-ray diffraction and X-ray fluorescence to identify a pool of guanine distinct from crystalline guanine and suggest that this material arises from vesicles whose elongation matches that expected of a growing crystal. Vignolini et al. determined that the blue coloration of Pollia condensata fruit is created by the combined action of three cell layers with different functions: the first reflects circular polarized light using a multilayered cell wall, the second layer absorbs the transmitted light using tannins, and the last layer backscatters the remaining light to the tannin layer. The study also showed the surprising presence of both right- and left-handed helicoidal microfibrils in the same tissue. 